\title{
Collision of solitons for a nonhomogeneous version of the $\mathrm{KdV}$ equation
}

\author{
George Omel'yanov*
}

\begin{abstract}
We consider KdV-type equations with $C^{1}$ nonhomogeneous nonlinearities and small dispersion $\varepsilon$. The first result consists of the conclusion that, in the leading term with respect to $\varepsilon$, the solitary waves in this model interact like KdV solitons. Next, it turned out that there exists a very interesting scenario of instability in which the short-wave soliton remains stable whereas a small long-wave part, generated by perturbations of original equation, turns to be unstable, growing and destroying the leading term. At the same time, such perturbation can eliminate the collision of solitons.
\end{abstract}

Key words: KdV-type equation, soliton, interaction, weak asymptotics method 2010 Mathematics Subject Classification: 35Q53, 35D30

\section{Introduction}

We consider a generalization of the KdV equation of the form:

$$
\frac{\partial u}{\partial t}+\frac{\partial g^{\prime}(u)}{\partial x}+\varepsilon^{2} \frac{\partial^{3} u}{\partial x^{3}}=0, x \in \mathbb{R}^{1}, t>0
$$

where $g^{\prime}(u) \stackrel{\text { def }}{=} \partial g / \partial u \in C^{1}$ is a real-valued function (for more detail see below) and $\varepsilon<<1$ is a small parameter. Such equations describe nonlinear wave phenomena in plasma physics. In particular, for some specific

*Universidad de Sonora, Rosales y Encinas s/n, 83000, Hermosillo, Sonora, México, omel@mat.uson.mx 
plasma states, ion-acoustic or dust-acoustic phenomena can be described by the KdV-type equation (1) with nonlinearities $g^{\prime}(u)=\alpha u^{3 / 2}+\beta u^{2}$ or $g^{\prime}(u)=\alpha u^{2}+\beta u^{3}, \alpha, \beta=$ const ([1] - [3]). To simplify the situation we restrict ourselves by non-negative $u$. Moreover, we assume that uniformly in $u \geq 0$

$$
c_{1} u^{1+\delta_{1}} \leq g^{\prime}(u) \leq c_{2} u^{5-\delta_{2}},
$$

where $c_{i}, \delta_{i}$ are positive constants. These restrictions imply for $\varepsilon=$ const both the solvability of the Cauchy problem for (1) and the solution stability with respect to initial data (see $[4,5])$.

For homogenous case $g^{\prime}(u)=u^{\kappa}, \kappa>1$, it is easy to find explicit solitary wave solutions (see below). Moreover, as it is well known nowadays, the solitons interact elastically in the integrable case $(\kappa=2$ and 3$)$. Almost the same is true for nonintegrable homogenous case: the solitary waves interact elastically in the principal term in an asymptotic sense, whereas the nonintegrability implies the appearance of small radiation-type corrections ([6] [12]). At the same time, the character of the solitary wave collision remains unknown for arbitrary nonlinearity. The same is true for the solitary wave stability with respect to right-hand side perturbations. Our aim is to consider these open problems.

The contents of the paper is the following: in Section 2 we find a class of nonlinearities which admits soliton type solutions, in Section 3 we demonstrate the elastic (in the leading asymptotic term) scenario of two soliton collision, and in the last section we describe the evolution of distorted solitons.

\section{Solitary wave solution}

Before the search of admissible nonlinearities we should determine the type of solitary waves which will be under consideration.

Definition 1. A function

$$
u=A \omega(\beta(x-V t) / \varepsilon, A)
$$

is called "soliton type solitary wave" if $\omega(\eta, \cdot) \in \mathcal{C}^{\infty}\left(\mathbb{R}^{1}\right)$ is an even function such that $\omega(0, \cdot)=1, \omega(\eta, \cdot)<1$ for $\eta \neq 0$, and $\partial^{2} \omega /\left.\partial \eta^{2}\right|_{\eta=0}<0$. Moreover, we assume that

$$
\omega(\eta, \cdot) \rightarrow 0 \quad \text { as } \quad \eta \rightarrow \pm \infty
$$


with an exponential rate. Next, we suppose that $\beta=\beta(A), V=V(A)$, $\omega=\omega(\cdot, A)$ are $C^{1}$ functions uniformly in the parameter $A>0$, and

$$
\partial \omega(\eta, A) / \partial A \rightarrow 0 \quad \text { as } \quad \eta \rightarrow 0 \quad \text { or } \quad \eta \rightarrow \pm \infty .
$$

Theorem 1. Let $g(u) \in \mathcal{C}^{2}(u \geq 0) \cap \mathcal{C}^{\infty}(u>0)$ satisfy (2) and be such that

$$
g(u)=u^{2} g_{1}(u)
$$

where the Hölder continuous function $g_{1}$ satisfies the conditions:

$$
g_{1}(0)=0, \quad g_{1}(u)>0 \quad \text { and } \quad g_{1}^{\prime}(u)>0 \quad \text { for } \quad u>0 .
$$

Then the equation (1) has a soliton type solitary wave solution.

To prove the statement it is enough to substitute the desired form (3) into the equation (1) and integrate it using the condition (4). We obtain

$$
-V \omega+\frac{g^{\prime}(A \omega)}{A}+\beta^{2} \frac{d^{2} \omega}{d \eta^{2}}=0
$$

which implies

$$
\frac{\beta^{2}}{V}\left(\frac{d \omega}{d \eta}\right)^{2}=\omega^{2}\left(1-\frac{2 g_{1}(A)}{V} G(\omega, A)\right), \quad G(\omega, A) \stackrel{\text { def }}{=} g_{1}(A \omega) / g_{1}(A) .
$$

Next, $\omega$ can be a smooth even function if and only if $\partial \omega /\left.\partial \eta\right|_{\eta=0}=0$. This and the normalization condition $\omega(0, \cdot)=1$ imply the equality

$$
V=2 g_{1}(A)
$$

In order to simplify the equation we define the free parameter $\beta$,

$$
\beta^{2}=V
$$

and obtain the final version of the equation for $\omega$

$$
\frac{d \omega}{d \eta}= \pm \omega \sqrt{1-G(\omega, A)}
$$

where the sign should be - for $\eta>0$ and + for $\eta<0$. 
To complete the proof we analyze the implicit representation of $\omega$ which corresponds to (12):

$$
\eta=\int_{\omega}^{1} \frac{d z}{z \sqrt{1-G(z, A)}} \text { for } \quad \eta \geq 0
$$

By virtue of (7) $\omega \sim 1-c_{1} \eta^{2}$ for $\eta \rightarrow 0$ and $\omega \sim \exp \left(-c_{2} \eta\right)$ for $\eta \rightarrow \infty$. Next, differentiating the both parts of (13) with respect to $A$, we obtain

$$
\frac{\partial \omega}{\partial A}=\frac{\omega \sqrt{1-G(\omega, A)}}{2 g_{1}^{2}(A)} \int_{\omega}^{1} \frac{z g_{1}^{\prime}(A z) g_{1}(A)-g_{1}^{\prime}(A) g_{1}(A z)}{z(1-G(z, A))^{3 / 2}} d z .
$$

Using (7) and this representation one easily verifies that $\partial \omega / \partial A \in C^{1}$ and tends to zero as $\eta \rightarrow \infty$ and $\eta \rightarrow 0$.

Example. The function

$$
g_{1}(z)=\sum_{k=1}^{n} c_{k} z^{q_{k}}, \quad \delta_{1} \leq q_{1}<q_{2}<\cdots<q_{n}<4, \quad c_{k}>0
$$

satisfies the conditions (7). If $n=1$, then the solution of (12) does not depend on $A$ and has the form:

$$
\omega(\eta)=\left\{\cosh \left(q_{1} \eta / 2\right)\right\}^{-2 / q_{1}}, \quad V=2 c_{1} A^{q_{1}} .
$$

\section{Two-soliton asymptotic solution}

\subsection{Main definitions}

Obviously, there is not any hope to find both the exact multi-soliton solution to (1) and an asymptotics in the classical sense. So, we will construct a weak asymptotic solution. The Weak Asymptotics Method (see e.g. [6] - [15] and references therein) takes into account the fact that soliton-type solutions which are smooth for $\varepsilon>0$ become non-smooth in the limit as $\varepsilon \rightarrow 0$. Thus, it is possible to treat such solutions as a mapping $\mathcal{C}^{\infty}\left(0, T ; \mathcal{C}^{\infty}\left(\mathbb{R}_{x}^{1}\right)\right)$ for $\varepsilon=$ const $>0$ and only as $\mathcal{C}\left(0, T ; \mathcal{D}^{\prime}\left(\mathbb{R}_{x}^{1}\right)\right)$ uniformly in $\varepsilon \geq 0$. Accordingly, the remainder should be small in the weak sense. The main advantage of the method is such that we can ignore the real shape of the colliding waves but 
look for (and find) exceptionally their main characteristics. For the solitons they are the amplitudes and trajectories of the waves.

Similarly the famous Whitham method we define a weak asymptotic solution as a function which satisfies (in a weak sense) some conservation laws, in fact two laws for the two-phase asymptotics. For the equation (1) let us write the first conservation laws in the differential form:

$$
\frac{\partial Q_{j}}{\partial t}+\frac{\partial P_{j}}{\partial x}=\varepsilon^{2} \frac{\partial^{3} R_{j}}{\partial x^{3}}, \quad j=1,2,
$$

where the first one is the equation (1) the same, namely

$$
\begin{gathered}
Q_{1}=u, \quad P_{1}=g^{\prime}(u), \quad Q_{2}=u^{2}, \quad P_{2}=-2 g_{2}(u)-3\left(\varepsilon u_{x}\right)^{2} \\
R_{1}=u, \quad R_{2}=u^{2}, \quad g_{2}(u)=g(u)-u g^{\prime}(u) .
\end{gathered}
$$

Next, we define the smallness in the weak sense:

Definition 2. A function $v(t, x, \varepsilon)$ is said to be of the value $O_{\mathcal{D}^{\prime}}\left(\varepsilon^{k}\right)$ if the relation

$$
\int_{-\infty}^{\infty} v(t, x, \varepsilon) \psi(x) d x=O\left(\varepsilon^{k}\right)
$$

holds uniformly in $t$ for any test function $\psi \in \mathcal{D}\left(\mathbb{R}_{x}^{1}\right)$. The right-hand side here is a $\mathcal{C}^{\infty}$-function for $\varepsilon=$ const $>0$ and a piecewise continuous function uniformly in $\varepsilon \geq 0$.

As it has been demonstrated in $[6,12]$, the correct definition of two-soliton asymptotics is the following:

Definition 3. A sequence $u(t, x, \varepsilon)$, belonging to $\mathcal{C}^{\infty}\left(0, T ; \mathcal{C}^{\infty}\left(\mathbb{R}_{x}^{1}\right)\right)$ for $\varepsilon=$ const $>0$ and belonging to $\mathcal{C}\left(0, T ; \mathcal{D}^{\prime}\left(\mathbb{R}_{x}^{1}\right)\right)$ uniformly in $\varepsilon$, is called a weak asymptotic $\bmod O_{\mathcal{D}^{\prime}}\left(\varepsilon^{2}\right)$ solution of (1) if the relations (17) hold uniformly in $t$ with the accuracy $O_{\mathcal{D}^{\prime}}\left(\varepsilon^{2}\right)$,

$$
\frac{\partial Q_{j}}{\partial t}+\frac{\partial P_{j}}{\partial x}=O_{\mathcal{D}^{\prime}}\left(\varepsilon^{2}\right), \quad j=1,2 .
$$

Let us consider the interaction of two solitary waves for the model (1) with the initial data

$$
\left.u\right|_{t=0}=\sum_{i=1}^{2} A_{i} \omega\left(\beta_{i} \frac{x-x_{i}^{0}}{\varepsilon}, A_{i}\right),
$$


where $A_{2}>A_{1}>0, x_{1}^{0}-x_{2}^{0}=$ const $>0$ and we assume the same relations between $A_{i}, \beta_{i}$ and $V_{i}$ as in (10). Obviously, the trajectories $x=V_{i} t+x_{i}^{0}$ have a joint point $x=x^{*}$ at a time instant $t=t^{*}$.

Following $[6,12]$, we write the asymptotic ansatz in the form:

$$
u=\sum_{i=1}^{2} G_{i}(\tau) \omega\left(\beta_{i} \frac{x-\varphi_{i}(t, \tau, \varepsilon)}{\varepsilon}, A_{i}\right), \quad G_{i}(\tau)=A_{i}+S_{i}(\tau) .
$$

Here $\varphi_{i}=\varphi_{i 0}(t)+\varepsilon \varphi_{i 1}(\tau)$, where $\varphi_{i 0}=V_{i} t+x_{i}^{0}$, are the trajectories of noninteracting solitary waves;

$$
\tau=\psi_{0}(t) / \varepsilon, \quad \psi_{0}(t)=\beta_{1}\left(\varphi_{20}(t)-\varphi_{10}(t)\right),
$$

denotes the "fast time"; the phase and amplitude corrections $\varphi_{i 1}, S_{i}$ are smooth functions such that

$$
\begin{gathered}
\varphi_{i 1}(\tau) \rightarrow 0 \text { as } \tau \rightarrow-\infty, \quad \varphi_{i 1}(\tau) \rightarrow \varphi_{i 1}^{\infty}=\text { const }_{i} \quad \text { as } \tau \rightarrow+\infty \\
S_{i}(\tau) \rightarrow 0 \quad \text { as } \tau \rightarrow \pm \infty
\end{gathered}
$$

with an exponential rate.

\subsection{Asymptotic construction}

To construct the asymptotics we should calculate the weak expansions of the terms from the left-hand sides of the relations (20). It is easy to check that

$$
u=\varepsilon \sum_{i=1}^{2} a_{1, i} \frac{G_{i}}{\beta_{i}} \delta\left(x-\varphi_{i}\right)+O_{\mathcal{D}^{\prime}}\left(\varepsilon^{3}\right),
$$

where $\delta(x)$ is the Dirac delta-function. Here and in what follows we use the notation

$$
a_{k, i} \stackrel{\text { def }}{=} \int_{-\infty}^{\infty}\left(\omega\left(\eta, A_{i}\right)\right)^{k} d \eta, \quad k>0, \quad a_{2, i}^{\prime} \stackrel{\text { def }}{=} \int_{-\infty}^{\infty}\left(\omega^{\prime}\left(\eta, A_{i}\right)\right)^{2} d \eta .
$$

At the same time for any $F(u) \in C^{1}$

$$
\int_{-\infty}^{\infty} F\left(\sum_{i=1}^{2} G_{i} \omega\left(\beta_{i} \frac{x-\varphi_{i}}{\varepsilon}, A_{i}\right)\right) \psi(x) d x
$$




$$
\begin{aligned}
& =\varepsilon \sum_{i=1}^{2} \frac{1}{\beta_{i}} \int_{-\infty}^{\infty} F\left(A_{i} \omega\left(\eta, A_{i}\right)\right) \psi\left(\varphi_{i}+\varepsilon \frac{\eta}{\beta_{i}}\right) d \eta+\frac{\varepsilon}{\beta_{2}} \int_{-\infty}^{\infty}\left\{F \left(G_{1} \omega\left(\eta_{12}, A_{1}\right)\right.\right. \\
& \left.\left.+G_{2} \omega\left(\eta, A_{2}\right)\right)-F\left(A_{1} \omega\left(\eta_{12}, A_{1}\right)\right)-F\left(A_{2} \omega\left(\eta, A_{2}\right)\right)\right\} \psi\left(\varphi_{2}+\varepsilon \frac{\eta}{\beta_{2}}\right) d \eta
\end{aligned}
$$

where

$$
\left.\eta_{12}=\theta \eta-\sigma, \quad \sigma=\beta_{1}\left(\varphi_{1}-\varphi_{2}\right)\right) / \varepsilon, \quad \theta=\beta_{1} / \beta_{2} .
$$

We take into account that the second integrand in right-hand side (27) vanishes exponentially fast as $\left|\varphi_{1}-\varphi_{2}\right|$ grows, thus, its main contribution is at the point $x^{*}$. We write

$$
\varphi_{i 0}=x^{*}+V_{i}\left(t-t^{*}\right)=x^{*}+\varepsilon \frac{V_{i}}{\dot{\psi}_{0}} \tau \text { and } \varphi_{i}=x^{*}+\varepsilon \chi_{i},
$$

where $\dot{\psi}_{0}=\beta_{1}\left(V_{2}-V_{1}\right), \chi_{i}=V_{i} \tau / \dot{\psi}_{0}+\varphi_{i 1}$. It remains to apply the formula

$$
f(\tau) \delta\left(x-\varphi_{i}\right)=f(\tau) \delta\left(x-x^{*}\right)-\varepsilon \chi_{i} f(\tau) \delta^{\prime}\left(x-x^{*}\right)+O_{\mathcal{D}^{\prime}}\left(\varepsilon^{2}\right),
$$

which holds for each $\varphi_{i}$ of the form (29) with slowly increasing $\chi_{i}$ and for $f(\tau)$ from the Schwartz space. Moreover, the second term in the right-hand side of (30) is $O_{\mathcal{D}^{\prime}}(\varepsilon)$. Thus, under the assumptions (23), (24) we obtain the weak asymptotic expansion of $F(u)$ in the final form:

$$
F(u)=\varepsilon \sum_{i=1}^{2} F\left(A_{i}\right) \frac{a_{F, i}}{\beta_{i}} \delta\left(x-\varphi_{i}\right)+\varepsilon \frac{F\left(A_{2}\right)}{\beta_{2}} \mathfrak{R}_{F} \delta\left(x-x^{*}\right)+O_{\mathcal{D}^{\prime}}\left(\varepsilon^{2}\right),
$$

where

$$
\begin{aligned}
a_{F, i} & =F\left(A_{i}\right)^{-1} \int_{-\infty}^{\infty} F\left(A_{i} \omega\left(\eta, A_{i}\right)\right) d \eta \\
\mathfrak{R}_{F} & =F\left(A_{2}\right)^{-1} \int_{-\infty}^{\infty}\left\{F\left(G_{1} \omega\left(\eta_{12}, A_{1}\right)+G_{2} \omega\left(\eta, A_{2}\right)\right)\right. \\
& \left.-F\left(A_{1} \omega\left(\eta_{12}, A_{1}\right)\right)-F\left(A_{2} \omega\left(\eta, A_{2}\right)\right)\right\} d \eta .
\end{aligned}
$$

Note that to define $\partial u^{2} / \partial t \bmod O_{\mathcal{D}^{\prime}}\left(\varepsilon^{2}\right)$ it is necessary to calculate $u^{2}$ with the precision $O_{\mathcal{D}^{\prime}}\left(\varepsilon^{3}\right)$. Thus, transforming (25) with the help of (30) and using (31) with $F(u)=u^{2}$, we obtain modulo $O_{\mathcal{D}^{\prime}}\left(\varepsilon^{3}\right)$ :

$$
u=\varepsilon \sum_{i=1}^{2} a_{1, i} K_{i 0}^{(1)} \delta\left(x-\varphi_{i}\right)+\varepsilon \sum_{i=1}^{2} a_{1, i} K_{i 1}^{(1)}\left\{\delta\left(x-x^{*}\right)-\varepsilon \chi_{i} \delta^{\prime}\left(x-x^{*}\right)\right\}
$$




$$
\begin{aligned}
& u^{2}=\varepsilon \sum_{i=1}^{2} a_{2, i} K_{i 0}^{(2)} \delta\left(x-\varphi_{i}\right)+\varepsilon \sum_{i=1}^{2}\left\{a_{2, i} K_{i 1}^{(2)}+2 \tilde{a}_{2} \frac{G_{1} G_{2}}{\beta_{2}} R_{2}^{(0)}\right\} \delta\left(x-x^{*}\right) \\
& -\varepsilon^{2}\left\{\sum_{i=1}^{2} a_{2, i} K_{i 1}^{(2)} \chi_{i}+2 \tilde{a}_{2} \frac{G_{1} G_{2}}{\beta_{2}}\left(\chi_{2} R_{2}^{(0)}+\frac{1}{\beta_{2}} R_{2}^{(1)}\right)\right\} \delta^{\prime}\left(x-x^{*}\right)
\end{aligned}
$$

where

$$
\begin{aligned}
& K_{i}^{(n)}=\frac{G_{i}^{n}}{\beta_{i}}, \quad K_{i 0}^{(n)}=\frac{A_{i}^{n}}{\beta_{i}}, \quad K_{i 1}^{(n)}=K_{i}^{(n)}-K_{i 0}^{(n)}, \\
& R_{2}^{(i)}=\frac{1}{\tilde{a}_{2}} \int_{-\infty}^{\infty} \eta^{i} \omega\left(\eta_{12}, A_{1}\right) \omega\left(\eta, A_{2}\right) d \eta, \quad \tilde{a}_{2}=\sqrt{a_{2,1} a_{2,2}} .
\end{aligned}
$$

Calculating weak expansions for other terms from the Definition 3 and substituting them into (20) we obtain linear combinations of $\delta^{\prime}\left(x-\varphi_{i}\right), i=1,2$, $\delta\left(x-x^{*}\right)$, and $\delta^{\prime}\left(x-x^{*}\right)$ (see also [6, 7]). Therefore, we obtain:

system of algebraic equations

$$
\begin{aligned}
& a_{1, i} V_{i} K_{i 0}^{(1)}-a_{g^{\prime}, i} g^{\prime}\left(A_{i}\right) / \beta_{i}=0, \quad i=1,2, \\
& a_{2, i} V_{i} K_{i 0}^{(2)}+2 a_{g_{2}, i} g_{2}\left(A_{i}\right) / \beta_{i}+3 a_{2, i}^{\prime} \beta_{i}^{2} K_{i 0}^{(2)}=0, \quad i=1,2,
\end{aligned}
$$

system of functional equations

$$
\sum_{i=1}^{2} a_{1, i} K_{i 1}^{(1)}=0, \quad \sum_{i=1}^{2} a_{2, i} K_{i 1}^{(2)}+2 \tilde{a}_{2} \frac{G_{1} G_{2}}{\beta_{2}} R_{2}^{(0)}=0,
$$

and system of ordinary differential equations

$$
\begin{aligned}
\dot{\psi}_{0} \frac{d}{d \tau} \sum_{i=1}^{2} a_{1, i}\left\{K_{i 0}^{(1)} \varphi_{i 1}\right. & \left.+\chi_{i} K_{i 1}^{(1)}\right\}=f \\
\dot{\psi}_{0} \frac{d}{d \tau}\left\{\sum _ { i = 1 } ^ { 2 } a _ { 2 , i } \left(K_{i 0}^{(2)} \varphi_{i 1}\right.\right. & \left.+\chi_{i} K_{i 1}^{(2)}\right) \\
& \left.+2 \frac{\tilde{a}_{2}}{\beta_{2}} G_{1} G_{2}\left(\chi_{2} R_{2}^{(0)}+\frac{1}{\beta_{2}} R_{2}^{(1)}\right)\right\}=F
\end{aligned}
$$

where

$$
f=\frac{g^{\prime}\left(A_{2}\right)}{\beta_{2}} \mathfrak{R}_{g^{\prime}}, \quad \tilde{a}_{2}^{\prime}=\sqrt{a_{2,1}^{\prime} a_{2,2}^{\prime}}
$$




$$
F=-2 \frac{g_{2}\left(A_{2}\right)}{\beta_{2}} \mathfrak{R}_{g_{2}}-3\left\{\sum_{i=1}^{2} a_{2, i}^{\prime} \beta_{i}^{2} K_{i 1}^{(2)}+2 \tilde{a}_{2}^{\prime} \beta_{1} G_{1} G_{2} R_{2,1}^{(0)}\right\},
$$

and $R_{2,1}^{(0)}$ is of the form (36) for $R_{2}^{(0)}$ but with $\tilde{a}_{2}^{\prime}, \omega^{\prime}$ instead of $\tilde{a}_{2}, \omega$.

Lemma 1. The algebraic equations (37), (38) imply again the relations (10), (11) between $A_{i}, \beta_{i}$, and $V_{i}$.

Proof. Let us change $A \rightarrow A_{i}, V \rightarrow V_{i}, \beta \rightarrow \beta_{i}$ in (8) and integrate it with respect to $\eta$. Then we obtain the equality

$$
a_{1, i} A_{i} V_{i}=a_{g^{\prime}, i} g^{\prime}\left(A_{i}\right),
$$

which is equivalent to (37). Next, let us multiply the original third order ordinary equation for $\omega$ by $\omega$. Integrating we obtain the following alternative version of (8):

$$
V \omega^{2}+2 \frac{g_{2}(A \omega)}{A^{2}}=\beta^{2}\left\{\frac{d^{2}\left(\omega^{2}\right)}{d \eta^{2}}-3\left(\frac{d \omega}{d \eta}\right)^{2}\right\}
$$

Thus

$$
a_{2, i} V_{i}+2 a_{g_{2}, i} \frac{g_{2}\left(A_{i}\right)}{A_{i}^{2}}=-3 a_{2, i}^{\prime} \beta_{i}^{2} .
$$

At the same time, integration of (9) implies:

$$
a_{2, i} V_{i}-2 a_{g, i} \frac{g\left(A_{i}\right)}{A_{i}^{2}}=a_{2, i}^{\prime} \beta_{i}^{2} .
$$

Taking into account (45) we obtain the relation

$$
a_{g, i} g\left(A_{i}\right)=-a_{g_{2}, i} g_{2}\left(A_{i}\right)-2 a_{2, i}^{\prime} \beta_{i}^{2} A_{i}^{2},
$$

which implies that (38) is the result of the integration of (9) with respect to $\eta$. Now we square the both parts of (12) and integrate them:

$$
a_{2, i}^{\prime}=a_{2, i}-a_{g, i} .
$$

Substituting this into (46) we obtain the equality

$$
a_{2, i}\left(V_{i}-\beta_{i}^{2}\right)-a_{g, i} g_{1}\left(A_{i}\right)\left(2-\frac{\beta_{i}^{2}}{g_{1}\left(A_{i}\right)}\right)=0,
$$

which implies the relations (10). 


\subsection{Analysis of the model equations (39) - (41)}

It is easy to note that the system (39) is equivalent to the quadratic equation with coefficients which depend on the convolution $R_{2}^{(0)}=R_{2}^{(0)}(\sigma)$. To analyze the equation we assume:

$$
A_{i}>>1, \quad i=1,2, \quad \theta<<1 .
$$

Moreover, let the function $g_{1}$ be of the form (15). Then (10), (11) imply the relation

$$
A_{i}=c^{\prime} \beta_{i}^{q^{\prime}}\left(1+O\left(A_{i}^{q_{n-1}-q_{n}}\right)\right), \quad q^{\prime}=2 / q_{n}, c^{\prime}=\left(2 c_{n}\right)^{-1 / q_{n}}, i=1,2 .
$$

Let us define the following notation:

$$
\kappa_{i}=\frac{S_{i}}{\beta_{i}}\left(c^{\prime} \beta_{2}^{q^{\prime}-1}\right)^{-1}, \quad i=1,2 .
$$

Definition 4. A function $f(\tau, \theta)$ is said to be of the value $O_{S}\left(\theta^{k}\right)$ if there exists a function $s(\tau)$ from the Schwartz space such that the estimate

$$
|f(\tau, \theta)| \leq c \theta^{k}|s(\tau)|
$$

holds uniformly in $\tau$ for a constant $c>0$.

We note also that, under the condition (23), the convolution $R_{2}^{(0)} \rightarrow 0$ as $\tau \rightarrow \pm \infty$ with an exponential rate.

Assumptions (49) and (15) allow to prove the statement:

Lemma 2. Let the assumptions (15), (49) be satisfied. Then the algebraic equations (39) have a unique solution $S_{1}, S_{2}$ with the property (24). More in detail, for sufficiently small $\theta$ we find

$$
\kappa_{1}=\frac{\sqrt{\bar{a}_{2}}}{\bar{a}_{1}} \theta^{q^{\prime}} R_{2}^{(0)}+O_{S}\left(\theta^{2}+\theta^{2 q^{\prime}}\right), \quad \kappa_{2}=-\bar{a}_{1} \kappa_{1}, \quad \bar{a}_{i}=a_{i, 1} / a_{i, 2} .
$$

Now let us simplify the equations (40), (41). We note firstly that in view of the first equation (39) and the identity

$$
\beta_{1}\left(\chi_{1}-\chi_{2}\right)=\sigma
$$


one can eliminate $\chi_{i}$ from the left-hand side of (40), since

$$
\sum_{i=1}^{2} a_{1, i}\left\{K_{i 0}^{(1)} \varphi_{i 1}+\chi_{i} K_{i 1}^{(1)}\right\}=\sum_{i=1}^{2} a_{1, i} K_{i 0}^{(1)} \varphi_{i 1}+a_{1,1} \frac{\sigma}{\beta_{1}} K_{11}^{(1)} .
$$

Simplifying in the same manner the equation (41), we transform (40), (41) to the following form:

$$
\begin{aligned}
& \dot{\psi}_{0} \frac{d}{d \tau}\left\{\sum_{i=1}^{2} a_{1, i} K_{i 0}^{(1)} \varphi_{i 1}+a_{1,1} \frac{\sigma}{\beta_{1}} K_{11}^{(1)}\right\}=f, \\
& \dot{\psi}_{0} \frac{d}{d \tau}\left\{\sum_{i=1}^{2} a_{2, i} K_{i 0}^{(2)} \varphi_{i 1}+a_{2,1} \frac{\sigma}{\beta_{1}} K_{11}^{(2)}+2 \tilde{a}_{2} \theta K_{1}^{(1)} K_{2}^{(1)} R_{2}^{(1)}\right\}=F,
\end{aligned}
$$

where $f$ and $F$ are defined in (42), (43).

The second step is the elimination of $\varphi_{i 1}$ from the model system. To do it we divide $\sigma$ into the growing $\beta_{1}\left(V_{1}-V_{2}\right) \tau / \dot{\psi}_{0}=-\tau$ and the bounded (if the assumptions (23) are satisfied) $\tilde{\sigma}=\sigma+\tau$ parts. Since

$$
\varphi_{11}=\varphi_{21}+\tilde{\sigma} / \beta_{1}
$$

we obtain from $(55)$

$$
\dot{\psi}_{0} \frac{d}{d \tau}\left\{r_{1} \varphi_{21}+\frac{\sigma}{\beta_{1}} K_{1}^{(1)}\right\}=\frac{f}{a_{1,1}}-\frac{\dot{\psi}_{0}}{\beta_{1}} K_{10}^{(1)} .
$$

Here and in what follows we use the notation

$$
r_{j}=\sum_{i=1}^{2} \frac{a_{j, i}}{a_{j, 1}} K_{i 0}^{(j)} \quad \text { for } \quad j=1 \quad \text { and } \quad j=2 .
$$

Now, transforming (56) in the same manner and applying the first assumption (23) we pass to the problem:

$$
\frac{d}{d \tau} Q(\sigma)=\mathfrak{F}(\sigma),\left.\quad \frac{\sigma}{\tau}\right|_{\tau \rightarrow-\infty} \rightarrow-1
$$

where

$$
\begin{aligned}
& Q=\frac{\sigma}{\beta_{1}}\left\{K_{1}^{(2)}-\frac{r_{2}}{r_{1}} K_{1}^{(1)}\right\}+\frac{2}{\sqrt{\bar{a}_{2}}} K_{1}^{(1)} K_{2}^{(1)} R_{2}^{(1)}, \\
& \mathfrak{F}=-\frac{1}{\beta_{1}}\left\{K_{10}^{(2)}-\frac{r_{2}}{r_{1}} K_{10}^{(1)}\right\}+\frac{1}{\dot{\psi}_{0}}\left\{\frac{1}{a_{2,1}} F-\frac{r_{2}}{a_{1,1} r_{1}} f\right\} .
\end{aligned}
$$

Sufficiently simple analysis of the equation (60) implies the statement: 
Lemma 3. Under the assumptions (15), (49) $Q(\sigma) \in C^{2}\left(\mathbb{R}^{1}\right)$ and $\mathfrak{F}(\sigma) \in$ $C^{1}\left(\mathbb{R}^{1}\right)$. Moreover, the following relations hold:

$$
\begin{aligned}
& \frac{d Q}{d \sigma}=-\frac{A_{1} A_{2}}{\beta_{1}^{2}}\left\{\frac{\bar{a}_{2}}{\bar{a}_{1}}-\theta^{q^{\prime}}+O_{S}\left(\theta+\theta^{q^{\prime}}\right)\right\}, \\
& \mathfrak{F}=\frac{A_{1} A_{2}}{\beta_{1}^{2}}\left\{\frac{\bar{a}_{2}}{\bar{a}_{1}}-\theta^{q^{\prime}}+O_{S}\left(\theta+\theta^{q^{\prime}}\right)\right\} .
\end{aligned}
$$

The uniform in $\tau$ inequality $\mathfrak{F}>0$ and the exponential type behavior of $\mathfrak{F}$ and $Q$ imply the existence of the function $\sigma$ such that $\tilde{\sigma}=\sigma+\tau$ is bounded and tends to its limiting values with an exponential rate. This and the equalities (57), (58) justify the existence of the required phase corrections $\varphi_{i 1}$ with the property (23).

The main result of this section is the following:

Theorem 2. Let the assumptions (15), (49) be satisfied. Then the solitary wave collision in the problem (1), (21) preserves the elastic scenario with accuracy $O_{\mathcal{D}^{\prime}}\left(\varepsilon^{2}\right)$ in the sense of Definition 2. The weak asymptotic solution has the form (22).

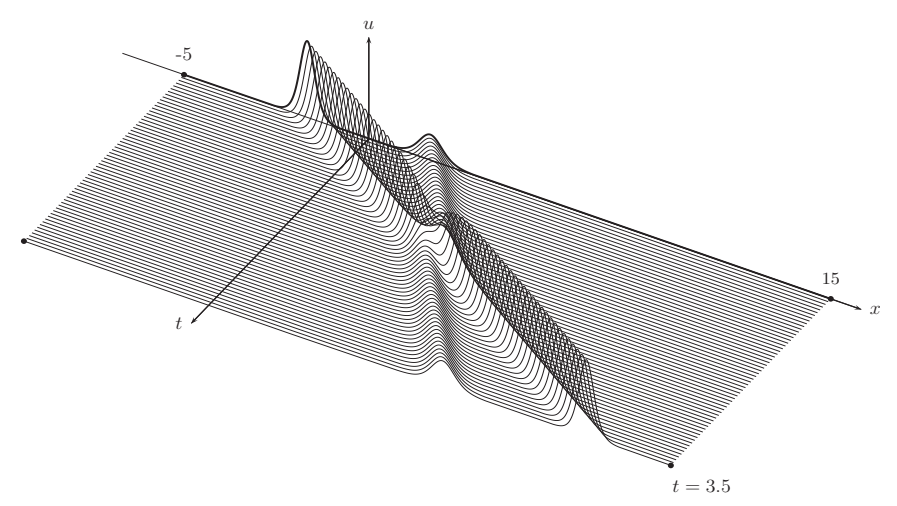

Figure 1: Evolution of two solitary waves with $A_{1}=1.5$ and $A_{2}=0.5$ for (1) with the nonlinearity $g^{\prime}=u^{3 / 2}+u^{2}$.

The next theorem allows us to treat the weak asymptotics (37) in the classical sense: 
Theorem 3. Let the assumptions (15), (49) be satisfied. Then the function $u$ of the form (22) is a weak asymptotic $\bmod O_{\mathcal{D}^{\prime}}\left(\varepsilon^{2}\right)$ solution of (1) if and only if $u$ satisfies the following conservation and balance laws:

$$
\begin{aligned}
& \frac{d}{d t} \int_{-\infty}^{\infty} u d x=0, \quad \frac{d}{d t} \int_{-\infty}^{\infty} u^{2} d x=0 \\
& \frac{d}{d t} \int_{-\infty}^{\infty} x u d x-\int_{-\infty}^{\infty} g^{\prime}(u) d x=0, \\
& \frac{d}{d t} \int_{-\infty}^{\infty} x u^{2} d x+2 \int_{-\infty}^{\infty} g_{2}(u) d x+3 \int_{-\infty}^{\infty}\left(\varepsilon \frac{\partial u}{\partial x}\right)^{2} d x=0 .
\end{aligned}
$$

To prove the Theorem 3 it is enough to rewrite the equalities (37) - (41) as integrals of the function (22) and its derivatives.

Results of direct numerical simulations confirm the traced asymptotic analysis. Figure 1 depicts the collision of two solitons for the nonlinearity $g^{\prime}=u^{3 / 2}+u^{2}$ in the case $\varepsilon=0.1$ (see also [11] for the nonlinearity $u^{3 / 2}$ ).

\section{Dynamics of perturbed solitary waves}

In this section we consider briefly the perturbed KdV-type equation (1),

$$
\frac{\partial u}{\partial t}+\frac{\partial g^{\prime}(u)}{\partial x}+\varepsilon^{2} \frac{\partial^{3} u}{\partial x^{3}}=F
$$

where $F=F\left(x, t, u, \varepsilon u_{x}, \varepsilon^{2} u_{x x}, \ldots\right) \in C^{\infty}$ is "small" for rapidly varying functions. We assume that $\left.F\right|_{u \equiv 0}=0$.

Let us construct firstly a self-similar one-phase asymptotic solution and discuss after that how to use this asymptotics for more realistic Cauchy data.

According to results $[10,12]$ to construct the leading term of the classical one-phase asymptotic solution it is enough to find the weak asymptotics. For the equation (66), instead of conservation laws, we have the balance laws

$$
\frac{\partial Q_{j}}{\partial t}+\frac{\partial P_{j}}{\partial x}+K_{j}=\varepsilon^{2} \frac{\partial^{3} R_{j}}{\partial x^{3}}, \quad j=1,2
$$

where $K_{1}=-F, K_{2}=-u F$, and all others terms are the same as in (18), (19).

By analogy with Definition 3 we write: 
Definition 5. Let a sequence $u(t, x, \varepsilon)$ belong to the same functional space as in Definition 3. Then $u(t, x, \varepsilon)$ is called a weak asymptotic mod $O_{\mathcal{D}^{\prime}}\left(\varepsilon^{\bar{q}}\right)$ solution of (66) if the relations

$$
\frac{\partial Q_{j}}{\partial t}+\frac{\partial P_{j}}{\partial x}+K_{j}=O_{\mathcal{D}^{\prime}}\left(\varepsilon^{\bar{q}}\right), \quad j=1,2
$$

hold uniformly in $t \in(0, T)$. Here $\bar{q}=\min \left\{1+q_{1}, 2\right\}$. form:

Combining the ideas of $[16,17]$ and $[10,12]$ we write the ansatz in the

$$
u=A \omega(\beta(x-\varphi(t)) / \varepsilon, A)+\varepsilon Y(\tau, t, x),
$$

where $A=A(t), \beta=\beta(t)$, and $Y$ is a smooth bounded function such that $Y(\tau, t, x) \rightarrow 0$ as $\tau \rightarrow+\infty$, and $Y(\tau, t, x) \rightarrow u^{-}(x, t)$ as $\tau \rightarrow-\infty$. Note that (69) can be treated as a "two-phase" asymptotics since

$$
u=\varepsilon a_{1} \frac{A}{\beta} \delta(x-\varphi(t))+\varepsilon u^{-}(x, t) H(\varphi(t)-x)+O_{\mathcal{D}^{\prime}}\left(\varepsilon^{2}\right),
$$

and the coefficient of the Heaviside function $H$ varies slowly.

We take into account the relations

$$
\begin{aligned}
& g^{\prime}(u)=\varepsilon \frac{a_{g^{\prime}}}{\beta} g^{\prime}(A) \delta(x-\varphi(t))+O_{\mathcal{D}^{\prime}}\left(\varepsilon^{\bar{q}}\right), \\
& F=\varepsilon \frac{a_{F_{0}}}{\beta} \bar{F} \delta(x-\varphi(t))+\left.\varepsilon F_{u}^{\prime}\right|_{u \equiv 0} u^{-}(x, t) H(\varphi(t)-x)+O_{\mathcal{D}^{\prime}}\left(\varepsilon^{2}\right),
\end{aligned}
$$

where we use the notation $(32), \bar{F}=F(\varphi, t, A, \beta A, \ldots), F_{0}=F(\varphi, t, A \omega$, $\left.A \beta \omega^{\prime}, A \beta^{2} \omega^{\prime \prime}, \ldots\right)$. Next, calculating others weak expansions and substituting them into (68) we obtain linear combinations of $\delta(x-\varphi), \delta^{\prime}(x-\varphi)$, and $H(\varphi-x)$. Therefore, we pass to the following system:

$$
\begin{aligned}
& a_{1} A \frac{d \varphi}{d t}=a_{g^{\prime}} g^{\prime}(A), \quad a_{2} \frac{d \varphi}{d t}+2 a_{g_{2}} \frac{g_{2}(A)}{A^{2}}+3 a_{2}^{\prime} \beta^{2}=0, \quad t>0, \\
& \frac{d}{d t}\left(a_{2} \frac{A^{2}}{\beta}\right)=2 a_{\omega F_{0}} \frac{A}{\beta} \bar{F}, \quad t>0, \\
& \frac{\partial u^{-}(x, t)}{\partial t}=F_{u}^{\prime}(x, t, 0, \ldots) u^{-}(x, t), \quad t>0, \quad x<\varphi(t), \\
& u^{-}(\varphi, t) \frac{d \varphi}{d t}+\frac{d}{d t}\left(a_{1} \frac{A}{\beta}\right)=\frac{a_{F_{0}}}{\beta} \bar{F} \quad t>0 .
\end{aligned}
$$


Lemma 1 implies that the equations (73) are equivalent to the equalities

$$
\frac{d \varphi}{d t}=\beta^{2}, \quad \beta^{2}=2 g_{1}(A) .
$$

Thus, the equations (74), (77) form the complete system to define $\varphi, A$, and $\beta$. We supply the equations by the Cauchy data:

$$
\left.\varphi\right|_{t=0}=\varphi^{0},\left.\quad A\right|_{t=0}=A^{0},
$$

where $\varphi^{0}$ and $A^{0}>0$ are arbitrary numbers.

Lemma 4. Under the assumptions (6), (15) the problem (74), (77), has the unique solution.

For the proof it is enough to demonstrate that the inequality

$$
I_{A}=\frac{d}{d A}\left(a_{2} \frac{A^{2}}{\beta}\right)>0
$$

holds uniformly in $A>0$. Indeed, using the representation (13) we obtain:

$$
\begin{aligned}
& I_{A}=\frac{A}{\sqrt{2}} \int_{0}^{1} \frac{z}{\left(g_{1}(A)-g_{1}(A z)\right)^{3 / 2}} J_{A}(z) d z, \\
& J_{A}(z)=4\left(g_{1}(A)-g_{1}(A z)\right)-A\left(g_{1}^{\prime}(A)-z g_{1}^{\prime}(A z)\right) .
\end{aligned}
$$

Furthermore, for the nonlinearity of the form (15) we have:

$g_{1}(A)>g_{1}(A z), J_{A}(z)=\sum_{k=1}^{n} c_{k}\left(4-q_{k}\right) A^{q_{k}}\left(1-z^{q_{k}}\right)>0$ uniformly in $z \in(0,1)$

since $q_{k} \in(0,4), k=1, \ldots n$.

Let us turn to the correction $u^{-}$. The equation (76) defines the boundary value of $u^{-}$on the curve $x=\varphi(t)$ for $t>0$. Thus, the problem to define the "tail" $u^{-}$consists of the equation (75) for $x<\varphi(t), t>0$, and the boundary condition:

$$
\left.u^{-}\right|_{x=\varphi(t)}=u_{b}^{-}(t) \quad \text { for } \quad t \geq 0,\left.\quad u^{-}\right|_{t=0}=u_{0}^{-}(x) \quad \text { for } \quad x \leq \varphi(0),
$$

where

$$
u_{b}^{-}(t)=\frac{1}{\beta^{3}}\left\{a_{F_{0}} \bar{F}-\beta \frac{d}{d t}\left(a_{1} \frac{A}{\beta}\right)\right\}
$$


and $u_{0}^{-}$is a smooth function such that $u_{0}^{-}(\varphi(0))=u_{b}^{-}(0)$. The solvability of this problem is obvious since $d \varphi / d t=\beta^{2}>0$. However, to obtain $u^{-} \geq 0$ we should suppose:

$$
a_{F_{0}} \bar{F} \geq \beta \frac{d}{d t}\left(a_{1} \frac{A}{\beta}\right), \quad u_{0}^{-} \geq 0 .
$$

This completes the asymptotic construction and we pass to the main result of the last section:

Theorem 4. Let the assumptions (6), (15) be satisfied. Then the function $u$ of the form (69) is a formal asymptotic mod $O\left(\varepsilon^{\bar{q}}\right)$ soliton-type solution of (66). The conditions (82) guarantee the fulfilment of the inequality $u \geq 0$.

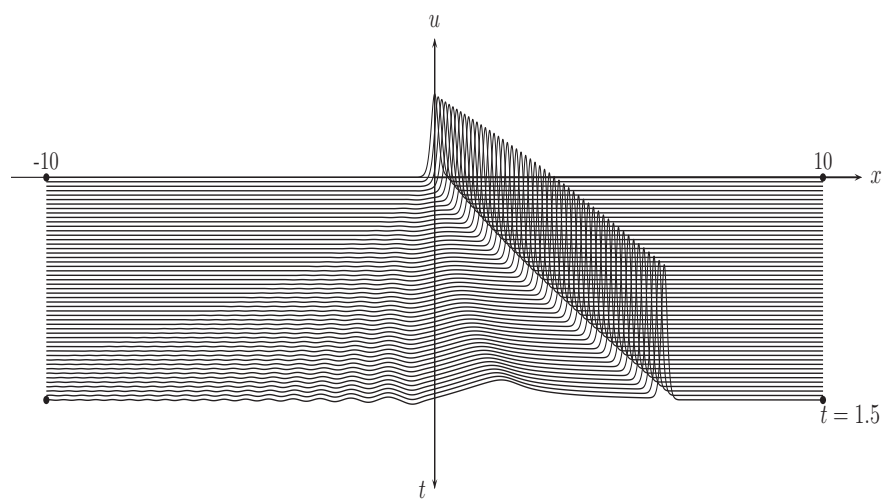

Figure 2: Perturbation of the solitary wave with $A(0)=4<A^{*}$

Let us stress finally that the self-similarity implies a special choice of the initial data. In particular, the initial function $Y(\tau, 0, x)$ should be of the special form

$$
Y(\tau, 0, x)=\left.\left\{u_{0}^{-}(x) \chi(\tau, t)+Z_{1}(\tau, t)+c_{1} \omega^{\prime}(\tau, A)\right\}\right|_{t=0}
$$

where $\chi(\tau, t)$ is a regularization of the Heaviside function, $Z_{1}(\tau, t)$ is a special function from the Schwartz space, and $c_{1}$ is arbitrary constant (see $\left.[16,17]\right)$. If it is violated and, for example,

$$
\left.u\right|_{t=0}=A(0) \omega(\beta(x-\varphi(0)) / \varepsilon, A(0)),
$$


then the perturbed soliton generates a rapidly oscillating tail of the amplitude $o(1)$ (the so called "radiation") instead of the smooth tail $\varepsilon u^{-}(x, t)$ (see [18] for the perturbed $\mathrm{KdV}$ equation and numerical results in [9]). However, $\varepsilon u^{-}(x, t)$ describes sufficiently well the tendency of the radiation amplitude behavior.

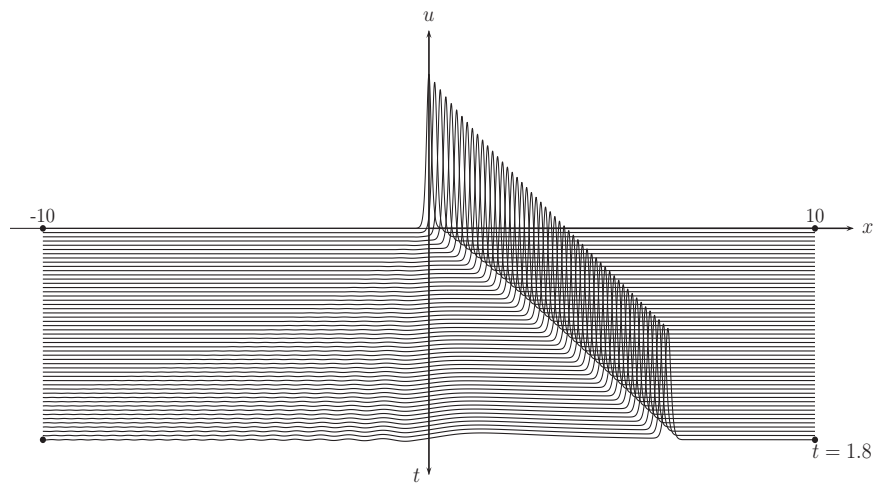

Figure 3: Perturbation of the solitary wave with $A(0)=6>A^{*}$

Example 1 [11]. Let $g^{\prime}(u)=u^{3 / 2}$ and let

$$
F=-\frac{\varepsilon}{2 b} \frac{\partial}{\partial x} u^{2}+\varepsilon \frac{b}{2} \frac{\partial}{\partial t}\left\{\frac{u^{2}}{2 b}-u^{3 / 2}-\varepsilon^{2} \frac{\partial^{2} u}{\partial x^{2}}-\frac{1}{2} \frac{\partial}{\partial t} \int_{-\infty}^{x} u d x^{\prime}\right\}, b=\text { const } .
$$

This right-hand side represents the remainder which was omitted in [1] in the process of the regular asymptotic construction. At the same time, for large $x$ and $t$ singular perturbations can appear and we should estimate the influence of (85) on the solitary wave. However it is easy to check that $a_{\omega F_{0}}=0$, thus $A=$ const. Consequently, $u^{-}(\varphi, t)=0$ and $u^{-}(x, t) \equiv 0$. This justifies the elimination of $F$ from the leading terms of the asymptotics.

Example 2. Let

$$
g^{\prime}(u)=u^{3 / 2}+u^{2}, \quad F=u(\mu-\nu u),
$$

where $\mu>0$ and $\nu>0$ are constants. Then the equation (74), supplied by the initial condition, takes the form:

$$
\frac{d A}{d t}=2 \nu a_{3} \frac{A^{2}}{\beta} I_{A}^{-1}\left(\frac{\mu a_{2}}{\nu a_{3}}-A\right),\left.\quad A\right|_{t=0}=A^{0}
$$




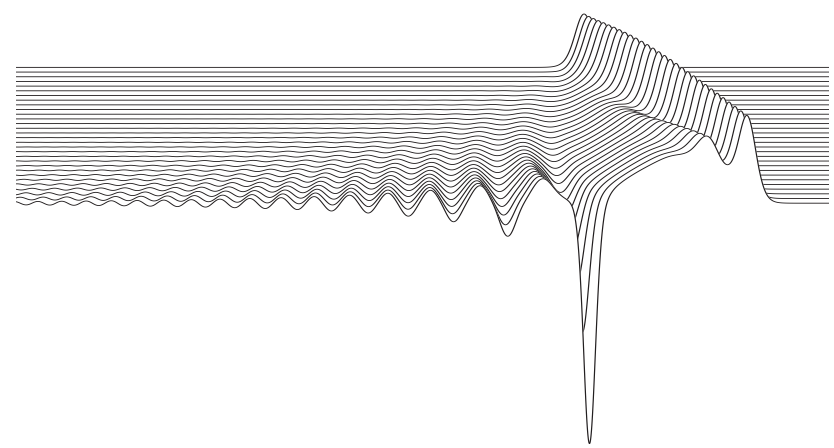

Figure 4: Destruction of the solitary wave.

where $I_{A}$ has the form (80) with $g_{1}(z)=2 \sqrt{z} / 5+z / 3$ and

$$
a_{k}=2 \int_{0}^{1} \frac{z^{k-1}}{\sqrt{1-G(z, A)}} d z .
$$

One can prove that there exists a critical amplitude $A^{*}=A^{*}(\mu / \nu)$ such that $A$ decreases to $A^{*}$ if $A^{0} \geq A^{*}$ or $A$ increases to $A^{*}$ if $A^{0} \leq A_{\alpha}^{*}$. In particular, $A^{*} \sim 4.9012$ for $\mu=2$ and $\nu=0.5$. Next, integrating the equation (77), we conclude that the curve $x=\varphi(t)$ tends to a straight line as $t \rightarrow \infty$. Thus, the solitary wave demonstrates a stable behavior.

Let us turn to the correction $u^{-}$. Preserving the term $O(\varepsilon)$, we write the equation (75) as following:

$$
\frac{\partial u^{-}}{\partial t}=u^{-}\left(\mu-\varepsilon \nu u^{-}\right)
$$

Thus, the amplitude of $u^{-}$increases exponentially fast with the rate $O(\mu)$. Moreover, it tends to the value $O(1 / \varepsilon)$, so that the correction $\varepsilon u^{-}$becomes of the same value as the leading term in a critical time $T^{*} \sim \mu^{-1} \ln (\mu /(\varepsilon \nu))$.

Results of numerical simulation confirm this analysis. Namely, we consider the Cauchy problem (66), (84), (86) with $\mu=2$ and $\nu=0.5$. Since the initial value does not include the correction of the form (83), the soliton correction is not a smooth tail $\varepsilon u^{-}(x, t)$, but the radiation. Note that the behavior of the correction's amplitude is explicitly the same as it been described above, see Figures 2 and 3 for a sufficiently small time instant. However, when the time tends to its critical value the soliton structure can be destroyed, see Fig. 4 for the case $g^{\prime}(u)=u^{3 / 2}[11]$. 


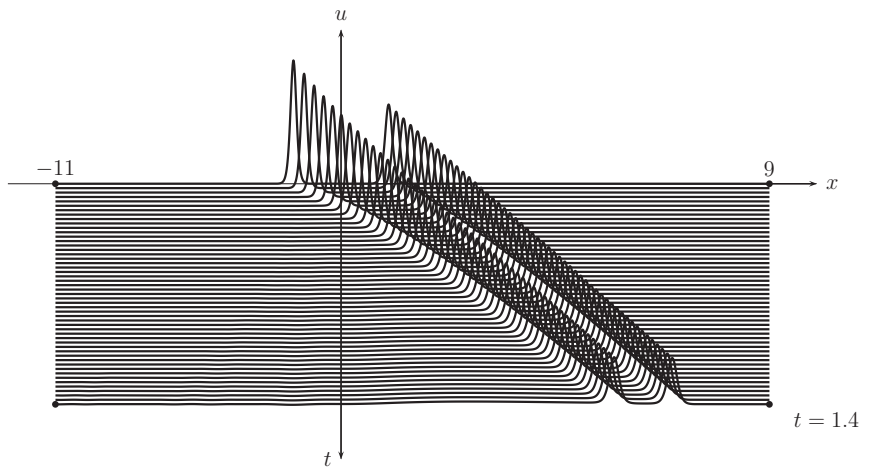

Figure 5: Evolution of the noninteracting solitary waves

\section{Conclusion}

In fact, the result that each equation from the family (1) preserves the KdVtype scenario of soliton interaction was rather expected. It was found much more interesting to consider the behavior of perturbed solitary waves. It turned out that there exists a class of perturbations which provoke a very interesting scenario of instability development: a short solitary wave (with the wave-length $\sim \varepsilon$ ) varies its parameters, remaining stable but generating a long wave perturbation (with the wave-length $\sim \varepsilon^{\nu}, \nu<1$ ) of a small amplitude. Inversely, this perturbation turns out to be unstable, its amplitude increases and destroys the original soliton.

On the other hand, the rate of the perturbation growth can be slow (of the order $O(\mu)$ with $\mu<<1$ for the external force (86)), whereas the amplitudes of solitons tend to the same stationary value $A_{\alpha}^{*}$. So, for sufficiently small $\mu$ (for sufficiently large distances between the original positions of the solitons), the amplitudes can be almost of the same value before the collision of solitons, which prevents the intersection of the trajectories. In other words, the perturbation can eliminate the interaction between solitons. To illustrate the situation we refer to Figure 5, where the dynamics of two solitons is depicted for the problem (66), (84), (86) with $\mu=2, \nu=0.5$, and the original amplitudes $A_{2}(0)=7, A_{1}(0)=4.5$. 


\section{Acknowledgement}

The research was supported by SEP-CONACYT under grant 178690 (Mexico).

\section{References}

[1] H. Schamel, A modified Korteweg-de Vries equation for ion acoustic waves due to resonant electrons, J. Plasma Phys. 9 (1973) 377-387, DOI: 10.1017/S002237780000756X.

[2] Kimiaki Konno, Yoshi H. Ichikawa, A modified Korteweg de Vries equation for ion acoustic waves, J. Phys. Soc. Jpn. 37 (1974) 1631-1636, Available from: https://doi.org/10.1143/JPSJ.37.1631.

[3] O. Rahman, M.D.I. Bhuyan, M.M. Haider, J. Islam, Dust-acoustic solitary waves in an unmagnetized dusty plasma with arbitrarily charged dust fluid and trapped ion distribution, IJAA. 4(1) (2014) DOI: 10.4236/ijaa.2014.41011.

[4] A.V. Faminskii, Cauchy problem for the Korteweg-de Vries equation and its generalizations, JSM. 50(1) (1990) 1381-1420, Available from: https://link.springer.com/article/10.1007/BF01097030.

[5] J.L. Bona, P.E. Souganidis, W. Strauss, Stability and instability of solitary waves of Korteweg-de Vries type, Proc. R. Soc. A. 411(1841) (1987) 395-412, DOI: 10.1098/rspa.1987.0073.

[6] V.G. Danilov, G.A. Omel'yanov, Weak asymptotics method and the interaction of infinitely narrow delta-solitons, Nonlinear Anal Theory Methods Appl. 54 (2003) 773-799, Available from: https://doi.org/10.1016/S0362-546X(03)00104-4.

[7] V.G. Danilov, G.A. Omel'yanov, V.M. Shelkovich, Weak asymptotics method and interaction of nonlinear waves, in: M.V. Karasev (Ed.), Asymptotic methods for wave and quantum problems, AMS Trans., Ser. 2, 208, AMS, Providence, RI, 2003, pp. 33-164, Available from: https://goo.gl/aPPhqG. 
[8] M.G. Garcia Alvarado, G.A. Omel'yanov, Interaction of solitary waves for the generalized $\mathrm{KdV}$ equation, Commun Nonlinear Sci Numer Simul. 17(8) (2012) 3204-3218, Available from: https://doi.org/10.1016/j.cnsns.2011.12.001.

[9] M.G. Garcia Alvarado, G.A. Omel'yanov, Interaction of solitons and the effect of radiation for the generalized $\mathrm{KdV}$ equation, Commun Nonlinear Sci Numer Simul. 19(8) (2014) 2724-2733, Available from: https://doi.org/10.1016/j.cnsns.2013.06.024.

[10] G.A. Omel'yanov, Soliton -type asymptotics for non-integrable equations: a survey, Math Methods Appl. 38(10) (2014) 2062-2071, DOI: 10.1002/mma.3198 2014.

[11] G.A. Omel'yanov, M.A. Valdez-Grijalva, Asymptotics for a $C^{1}$-version of the KdV equation, Nonl Phen Compl Sys. 17(2) (2014) 106-115, Available from: http://elib.bsu.by/handle/123456789/116849.

[12] G.A. Omel'yanov, Propagation and interaction of solitons for nonintegrable equations, RUSS J MATH PHYS. 23(2) (2016) 225-243, DOI:10.1134/S1061920816020084.

[13] V. Danilov, D. Mitrovic, Shock wave formation process for a multidimensional scalar conservation law, Quart. Appl. Math. 69(4) (2011) 613-63, Available from: https://doi.org/10.1090/S0033-569X-2011-01234-9.

[14] H. Kalisch, D. Mitrovic, Singular solutions of a fully nonlinear $2 \times 2$ system of conservation laws, Proc. Edinb. Math. Soc. (2). 55 (2012) 711-729, DOI: https://doi.org/10.1017/S0013091512000065 .

[15] E. Abreu, M. Colombeau, E. Panov, Weak asymptotic methods for scalar equations and systems. J Math Anal Appl. 444(2) (2016) 12031232, Available from: https://doi.org/10.1016/j.jmaa.2016.06.047.

[16] V.P. Maslov, G.A. Omel'yanov, Asymptotic soliton-form solutions of equations with small dispersion, RUSS MATH SURV. 36(3) (1981) 73149, Available from: http://iopscience.iop.org/0036-0279/36/3/R02.

[17] V.P. Maslov, G.A. Omel'yanov, Geometric asymptotics for nonlinear PDE, AMS, MMONO 202, Providence, RI, 2001. 
[18] L. Kalyakin, Perturbation of the Korteweg-de Vries soliton, Theoret. Math. Phys. 92 (1992) 736-747, Available from: https://goo.gl/aPPhqG. 